\title{
Chemical Descriptors of Yttria Stabilised Zirconia at Low Defect Concentration: an ab initio Study
}

Michael A. Parkes ${ }^{a, b}$, Keith Refson ${ }^{c}$, Mayeul d'Avezac ${ }^{d}$, Gregory J.Offer $^{e}$, Nigel P. Brandon ${ }^{a}$ and Nicholas M. Harrison ${ }^{b}$

${ }^{\mathrm{a}}$ Department of Earth Science and Engineering, Imperial College London,

SW7 2BP, UK

${ }^{b}$ Thomas Young Centre, Department of Chemistry, Imperial College London, London,

SW7 2AZ, UK

${ }^{\mathrm{c}}$ Department of Physics, Royal Holloway, University of London, Egham,

TW20 0EX, UK

${ }^{\mathrm{d}}$ Research Software Development Team, Research Computing and Facilitating Services,

University College London, London WC1E 6BT, UK

${ }^{\mathrm{e}}$ Department of Mechanical Engineering, Imperial College London,

SW7 2AZ, UK 
Keywords: solid oxide fuel cell, yttria stabilised zirconia, cubic zirconia, bixbyite yttria, $a b$ initio, density functional theory, empirical potential, Born-Mayer-Huggins, point charge model, phonons, harmonic approximation.

\section{Abstract}

Yttria stabilised zirconia (YSZ) is an important oxide ion conductor with applications in solid oxide fuel cells (SOFCs) and oxygen sensing devices. Doping the cubic phase of zirconia (c$\left.\mathrm{ZrO}_{2}\right)$ with yttria $\left(\mathrm{Y}_{2} \mathrm{O}_{3}\right)$ is isoelectronic, as two $\mathrm{Zr}^{4+}$ ions are replaced by two $\mathrm{Y}^{3+}$ ions, plus a charge compensating oxygen vacancy $\left(\mathrm{O}_{\mathrm{vac}}\right)$. Typical doping concentrations include 3, 8, 10 and $12 \mathrm{~mol} \%$. For these concentrations, and all below $40 \mathrm{~mol} \%$, no phase with long range order has been observed in either X-ray or neutron diffraction experiments. The prediction of local defect structure and the interaction between defects is therefore of great interest. This has not been possible to date as the number of possible defect topologies is very large and to perform reliable total energy calculations for all of them would be prohibitively expensive. Previous theoretical studies have only considered a selection of representative structures. In this study, a comprehensive search for low energy defect structures using a combined classical modelling and density functional theory approach is used to identify the low energy isolated defect structures at the dilute limit, $3.2 \mathrm{~mol} \%$. Through analysis of energetics computed using the best available Born-Mayer-Huggins empirical potential model, a point charge model, DFT, and a local strain energy estimated in the harmonic approximation, the main chemical and physical descriptors that correlate to the low energy DFT structures are discussed. It is found that the empirical potential model reproduces a general trend of increasing DFT energetics across a series of locally strain relaxed structures, but is unreliable both in predicting some incorrect low energy structures, and 
in finding some meta-stable structures to be unstable. A better predictor of low energy defect structures is found to be the total electrostatic energy of a simple point charge model calculated at the unrelaxed geometries of the defects. In addition, the strain relaxation energy is estimated effectively in the harmonic approximation to the imaginary phonon modes of undoped c- $\mathrm{ZrO}_{2}$, but is found to be unimportant in determining the low energy defect structures. These results allow us to propose a set of easily computed descriptors that can be used to identify the low energy YSZ defect structures, negating the combinatorial complexity and number of defect structures that need to be considered.

\section{Introduction}

Yttria stabilised zirconia (YSZ) is an oxide ion conductor at elevated temperatures. The $\left(\mathrm{Y}_{2} \mathrm{O}_{3}\right)_{0.1}\left(\mathrm{ZrO}_{2}\right)_{0.9}$ composition, conventionally referred to as $10 \mathrm{~mol} \% \mathrm{Y}_{2} \mathrm{O}_{3}$, has an ionic conductivity of approximately $0.03 \mathrm{Scm}^{-1}$ at $1000^{\circ} \mathrm{C}^{1}$. In addition to high anionic conductivity, it has other properties that make it an appropriate electrolyte for solid oxide fuel cells (SOFCs). These include its mechanical and chemical stability towards other SOFC components including nickel $(\mathrm{Ni})$ and lanthanum manganese oxide $\left(\mathrm{LaMnO}_{3}\right)$, high electronic resistivity, and its relative abundance and low cost when compared to other oxide ion conducting materials ${ }^{2}$. YSZ is a key component of the SOFC anode where it forms an interface with a catalytic metal and a gas phase. The interface region is referred to as the anode triple phase boundary (TPB) $)^{3-8}$. There is a growing interest in developing predictive models of the physical and chemical properties of YSZ and its surfaces in order to model the chemistry occurring at the anode TPB. A detailed knowledge of the surface chemistry is, however, currently inhibited by a poor understanding of 
the distribution of, and local atomistic structure surrounding the $\mathrm{Y}$ and $\mathrm{O}_{\text {vac }}$ dopants in the bulk crystal and at its surfaces ${ }^{9}$.

$\mathrm{ZrO}_{2}$ exhibits three main crystal structures under standard pressure ${ }^{10}$. At room temperature it adopts a monoclinic (m) structure which upon heating undergoes phase transitions to tetragonal (t) and cubic (c) phases at $1170^{\circ} \mathrm{C}$ and $2370^{\circ} \mathrm{C}$ respectively. The cubic phase exhibits the highest oxide ion $\left(\mathrm{O}^{2-}\right)$ conductivity making it the most technologically useful. Doping with $\mathrm{Y}_{2} \mathrm{O}_{3}$ is used to stabilise the cubic phase and thereby reduce the temperature at which the $\mathrm{t} \rightarrow \mathrm{c}$ phase transition occurs. Typical doping concentrations include 3, 8, 10 and $12 \mathrm{~mol} \%$. The exact location of the phase boundary between cubic and tetragonal transition as a function of $\mathrm{Y}_{2} \mathrm{O}_{3}$ concentration is still debated, ${ }^{11}$ however X-ray diffraction data suggests that $8 \mathrm{~mol} \%$ is the minimum concentration at which the cubic phase is stable at room temperature ${ }^{12-14}$. At $40 \mathrm{~mol} \%$, the ordered compound $\mathrm{Zr}_{3} \mathrm{Y}_{4} \mathrm{O}_{12}$ is formed ${ }^{15-17}$.

Previous calculations of the phonon spectrum using the generalised gradient approximation (GGA) to density functional theory (DFT) report an imaginary mode ${ }^{18-21}$ of frequency $i 195 \mathrm{~cm}^{-1}$ at the X-point of the first Brillouin zone corresponding to the low-temperature instability of the cubic phase $\mathrm{e}^{20}$. The eigenvector of this mode involves displacements of oxygen anions along the $\left\langle\begin{array}{lll}1 & 0 & 0\end{array}\right\rangle$ direction breaking the cubic symmetry which, upon full relaxation of the cell and internal coordinates, results in the observed tetragonal phase $\mathrm{e}^{22}$. Other first principles DFT calculations, for some representative defect structures, have suggested that the X-point imaginary phonon is stabilised as the $\mathrm{Y}_{2} \mathrm{O}_{3}$ concentration is increased from 0 to $10.4 \mathrm{~mol} \%{ }^{23}$.

Doping the cubic phase with $\mathrm{Y}_{2} \mathrm{O}_{3}$ is isoelectronic as two $\mathrm{Zr}^{4+}$ ions are replaced by two $\mathrm{Y}^{3+}$ ions, plus an oxygen vacancy $\left(\mathrm{O}_{\mathrm{vac}}\right)$ to maintain charge neutrality. In Kröger-Vink notation this is: 


$$
Y_{2} \mathrm{O}_{3} \stackrel{\mathrm{ZrO}_{2}}{\longrightarrow} 2 Y_{Z r}^{\prime}+V_{o}+3 O_{o}^{X}
$$

Where $Y_{Z r}^{\prime}$ indicates a yttrium ion on a zirconium lattice site with a negative charge, $V_{o}$. indicates a vacancy on an oxygen lattice site with +2 charge, and $30_{o}^{X}$ indicates lattice oxygen ions.

Because the $\mathrm{O}_{\text {vac }}$ facilitates the conduction of $\mathrm{O}^{2-}$ ions in the lattice, the conductivity depends on the $\mathrm{Y}_{2} \mathrm{O}_{3}$ concentration. For low concentrations the conductivity rises rapidly with the number of defects and reaches a maximum at $8 \mathrm{~mol} \%{ }^{24}$. It has been argued that inter-defect interactions reduce the conductivity at higher concentrations because of the increased electrostatic interaction between dopant cations and vacancies ${ }^{1,2}$.

The local atomistic structure and distribution of the dopants in YSZ has therefore been the topic of much theoretical and experimental research. Based on diffuse neutron scattering it has been reported that $\mathrm{O}_{\text {vacs }}$ preferentially associate in pairs along $\left\langle\begin{array}{lll}1 & 1 & 1\end{array}\right\rangle$ directions at dopant concentrations between 10 and $24 \mathrm{~mol} \%$ without forming a phase with long range order ${ }^{15,25,26}$. Theoretical evidence for the short range ordering of vacancies in $\left\langle\begin{array}{llll}1 & 1 & 1\end{array}\right\rangle$ directions has also been provided by DFT calculations ${ }^{16,17,23,27}$. In addition the electron paramagnetic resonance (EPR) spectrum of YSZ contains a prominent trigonal ( $T$ centre) peak in samples that have been chemically reduced or exposed to ionizing radiation ${ }^{28-31}$. This peak has been assigned to a vacancy-vacancy pair lying in a $\left\langle\begin{array}{llll}1 & 1 & 1\end{array}\right\rangle$ direction neighbouring a $\mathrm{Ti}^{3+}$ impurity ${ }^{27,32,33}$.

The O-coordination of metal ions within YSZ has also been studied. Early work suggested that a so called nearest neighbour $(\mathrm{NN})$ type defect structure, with the $\mathrm{O}_{\mathrm{vac}}$ in the first coordination shell of $\mathrm{Y}^{3+}$, is prevalent on the basis of neutron diffraction, X-ray absorption spectroscopy 
(EXAFS) and a simple point charge model of the ionic interactions ${ }^{34-37}$. In this model, $\mathrm{O}_{\text {vacs }}$ and $\mathrm{Y}^{3+}$ ions have effective charges, relative to the lattice ions, of +2 and -1 respectively so that their effective interaction is attractive. Structures with the $\mathrm{O}_{\text {vac }}$ in the first O-coordination sphere of yttrium will therefore have the lowest energies ${ }^{34-37}$. More recent work supports an alternative next nearest neighbour (NNN) model of defect structures, where the $\mathrm{O}_{\text {vac }}$ is found in the first $\mathrm{O}$ coordination sphere of $\mathrm{Zr}^{4+}$ and the second O-coordination sphere of $\mathrm{Y}^{3+}$ (examples of these structures are depicted in Figures 4 and 8). EXAFS $^{38-40}$ and solid state ${ }^{89}$ Y-MAS-NMR ${ }^{41}$ experiments, DFT total energy calculations ${ }^{42}$ and thermodynamics based on cluster expansion ${ }^{43}$, have established that, in general, $\mathrm{Y}^{3+}$ ions prefer 8-fold $\mathrm{O}$-coordination while $\mathrm{Zr}^{4+}$ ions have 7fold O-coordination. This tendency is also apparent in the low temperature monoclinic ground state of zirconia ${ }^{42}$. The ${ }^{89}$ Y-MAS-NMR data is particularly clear, as the observation of an intense 8-fold O-coordination ${ }^{89} \mathrm{Y}$ peak establishes that the $\mathrm{O}_{\mathrm{vac}}$ avoids the first coordination shell of $\mathrm{Y}^{3+}$ up to concentrations of around $12.5 \mathrm{~mol} \%$. This observation is inconsistent with the simple point charge model of the ionic interactions.

Many possible defect arrangements and orientations are consistent with the preference for $\mathrm{Zr}^{4+}$ to have 7 -fold and $\mathrm{Y}^{3+}$ to have 8-fold O-coordination. X-ray and neutron diffraction show that the defect structure of YSZ is highly disordered with multiple distinct defect clusters ${ }^{26}$, but the low contrast in the $\mathrm{X}$-ray and neutron scattering powers of $\mathrm{Zr}^{4+}$ and $\mathrm{Y}^{3+}$ ions give little information on the relative positions of the $\mathrm{Y}^{3+}$ and $\mathrm{O}_{\text {vac }}$ species or the local geometry of the dopant structures ${ }^{15}$.

When dopant $\mathrm{O}_{\text {vacs }}$ and cations are introduced onto the ideal fluorite lattice sites of c- $\mathrm{rO}_{2}$ without relaxation of the local geometry, the trend in the DFT formation energies is described well by a simple point charge model, and NN type defect structures are favoured ${ }^{17,23}$. Upon 
relaxation of the atomic positions within DFT, however, the NNN type defect structures are favoured at low defect concentrations. For the 6.7 and $10.4 \mathrm{~mol} \%$ concentrations significant relaxations occur as the $\mathrm{O}_{\text {vac }}$ undergoes barrier-less migrations (reconstructions) away from its starting location on the ideal c- $\mathrm{ZrO}_{2}$ lattice ${ }^{44}$ (an example is pictured in Figure 8). This instability of $\mathrm{O}_{\mathrm{vac}}$ geometries upon relaxation in DFT calculations is inconsistent with the point charge model and is also not reproduced faithfully by more sophisticated force-fields involving short range repulsions, Van der Waals forces, and ionic polarisation that have been fitted carefully to diffuse neutron scattering data ${ }^{44-46}$. The atomic positions and orientation of NNN type defects at low dopant concentrations appear to be governed by a combination of electrostatics and manybody strain elastic terms that have yet to be reduced to an analytic form ${ }^{1744}$. For high dopant concentrations $(>17 \mathrm{~mol} \%)$ and for the ordered compound $\mathrm{Zr}_{3} \mathrm{Y}_{4} \mathrm{O}_{12}(40 \mathrm{~mol} \%)$, the relative importance of these effects have been established ${ }^{16,17}$, however at the low dopant concentrations of 6.7 and $10.4 \mathrm{~mol} \%$, which are more relevant to oxide ion conductivity, the relative importance of these interactions is not clear, and it is not been possible to link the instability of structures to simple energetic or geometric parameters ${ }^{44}$.

In the absence of long range order at low dopant concentrations, identifying low energy defect topologies reliably is impossible without computing the total energy of every possible defect structure, a process that is infeasible with current implementations DFT. In the current work, we evaluate a set of easily computed descriptors that correlate to the final relaxed DFT energies of isolated defects modelled within bulk $\mathrm{c}-\mathrm{ZrO}_{2}$, at the dilute limit of $3.2 \mathrm{~mol} \%$. By studying the dilute limit we negate defect-defect interactions and the combinatorial complexity of higher dopant concentrations. At this concentration it is also possible to compute the energies of a complete set of symmetry inequivalent $3.2 \mathrm{~mol} \%$ YSZ structures using both an empirical force 
field $^{10,46-48}$ and DFT. We initially evaluate the ability of the Born-Mayer-Huggins (BMH) empirical force field to reproduce the trend in DFT energetics. It is found that the BMH empirical potential model reproduces a general trend of increasing DFT energetics across a series of locally strain relaxed structures, but is unreliable both in predicting some incorrect low energy structures, and in finding some meta-stable structures to be unstable. We also examine the use of electrostatic interactions, computed using formal charges in a point charge model, and the local strain energy estimated in the harmonic approximation, to predict trends in DFT energetics. We show that the relative formation energies of the relaxed DFT defect structures are correlated to a simple electrostatic energy and a strain contribution using a method similar to that used to describe clustering and relaxation in metallic alloys ${ }^{49}$. This allows us to provide reliable descriptors that correlate to the low energy DFT structures at the dilute limit. Finally, we evaluate why the best available BMH empirical force field is unreliable.

\section{Computational Details}

DFT calculations are performed within the plane-wave pseudopotential formalism as implemented in the CASTEP code $^{50}$. Empirical force field calculations are based on an energy expression of the Born-Mayer-Huggins form as implemented in the GULP code ${ }^{51}$.

DFT calculations were performed using the Perdew-Burke-Ernzerhof (PBE) GGA exchangecorrelation (XC) functional ${ }^{52}$. Atomic cores were replaced with ultrasoft pseudopotentials $(\mathrm{USPs})^{53}$ for which a kinetic energy cut off in the plane-wave expansion of $500 \mathrm{eV}$ converged the energy of the $\mathrm{c}-\mathrm{ZrO}_{2}$ phase to $0.3 \mathrm{meV}$ per formula unit. Phonon calculations were performed using density functional perturbation theory ${ }^{54}$ (DFPT) and norm-conserving pseudopotentials $(\mathrm{NCPs})^{55,56}$ with an energy cut off of $800 \mathrm{eV}$. Brillouin zone sampling was performed using a $3 \mathrm{x}$ 
$3 \times 3$ Monkhorst-Pack (MP) grid $^{57}$ in both the $2 \times 2 \times 2$ supercell of $\mathrm{c}-\mathrm{ZrO}_{2}$ and the primitive bixbyite cell of $\mathrm{Y}_{2} \mathrm{O}_{3}$. The self-consistent field (SCF) iterations were considered converged when the energy changed by less than $5 \times 10^{-9} \mathrm{eV}$ per cell for total energy calculations and $1 \times 10^{-10} \mathrm{eV}$ per cell for phonon calculations. Structural optimization was performed using the BroydenFletcher-Goldfarb-Shanno (BFGS) algorithm without symmetry constraints, with an atomic force convergence tolerance of $0.05 \mathrm{eV} / \AA^{-1}$. All defect structure calculations were performed at the zero-pressure PBE-DFT lattice constants of the parent materials to best model isolated defects at the dilute limit. These values are $10.22 \AA$ for the $2 \times 2 \times 2$ supercell of $\mathrm{c}-\mathrm{ZrO}_{2}$ and $10.65 \AA$ for the conventional cell of $\mathrm{Y}_{2} \mathrm{O}_{3}$. We also compute the bulk-modulus of the materials through fitting the Birch-Murnaghan equation of state to a set of cohesive energy - unit cell volume curves. We obtain a bulk modulus of $234 \mathrm{GPa}$ for c- $\mathrm{ZrO}_{2}$ and a bulk modulus of $144 \mathrm{GPa}$ for $\mathrm{Y}_{2} \mathrm{O}_{3}$. Both our lattice constants and bulk moduli are in excellent agreement with previous all electron linearised augmented plane-wave (LAPW) GGA-DFT calculations ${ }^{58,59}$. The PBE functional was used in the current study as it has been shown to successfully describe the parent compounds c- $\mathrm{ZrO}_{2}$, and $\mathrm{Y}_{2} \mathrm{O}_{3}$ in previous studies, and as a functional without empirical parameters, provides a consistent method for predicting defect energies ${ }^{58,59}$.

Computed phonon frequencies are sensitive to numerical approximations ${ }^{60}$ and therefore rigorous numerical tolerances were used in those calculations. Electron densities and pseudopotential augmentation charges were represented on an FFT grid one and a half times finer than that used to represent the Kohn-Sham orbitals in total energy calculations and two and a half times finer in phonon calculations. The implementation restriction to NCPs for phonon calculations potentially introduces some additional pseudopotential error. Comparison with the USPs yields an increase in the computed lattice constant of $\mathrm{c}-\mathrm{ZrO}_{2}$ of just $0.2 \%$, and a small 
change in the computed phonon frequencies, with a consequent error in calculated harmonic relaxation energies (plotted in Figure 5) of approximately $0.4 \mathrm{meV}$.

A Born-Mayer-Huggins (BMH) polarisable shell model based on formal ionic charges has been used by several authors to model defects in zirconia ${ }^{10,44-46,48}$. The form of the potential and the parameters are shown in equation 1 and Tables $1 \& 2$. The $\mathrm{Zr}-\mathrm{O}$ interaction parameters were determined by Dwivedi and Cormack ${ }^{46}$ by fitting a potential to the experimental lattice and dielectric constants of $\mathrm{t}-\mathrm{ZrO}_{2}{ }^{46}$. Those for $\mathrm{Y}-\mathrm{O}$ and $\mathrm{O}-\mathrm{O}$ were determined by Lewis and Catlow ${ }^{47}$. In addition to $\mathrm{t}-\mathrm{ZrO}_{2}$, the $\mathrm{Zr}-\mathrm{O}$ potentials have been shown to accurately reproduce the experimental lattice constants of stabilised $\mathrm{c}-\mathrm{ZrO}_{2}$, but not those of $\mathrm{m}-\mathrm{ZrO}_{2}$ which is unstable with respect to an orthorhombic phase ${ }^{10}$. The $\mathrm{Y}-\mathrm{O}$ potential accurately reproduces the experimental lattice constants of bixbyite $\mathrm{Y}_{2} \mathrm{O}_{3}{ }^{10}$. Calculations of $\mathrm{YSZ}$ defects using these potentials reproduce the fact that NNN type structures are more stable than NN type ${ }^{10,48}$.

$$
\varphi_{r}=\frac{q_{a} q_{b}}{r}+\operatorname{Aexp}^{(-r / \rho)}-\frac{B}{r^{6}}
$$

-equation 1

Table 1: YSZ Born-Mayer-Huggins potential parameters.

\begin{tabular}{|c|c|c|c|c|}
\hline \multicolumn{5}{|c|}{ Short Range Interactions } \\
\hline Interaction & $\mathrm{A} / \mathrm{eV}$ & $\rho / \AA$ & $\mathrm{B} / \mathrm{eV} \AA^{6}$ & Cut off / $\AA$ \\
\hline $\mathrm{Zr}^{4+} \ldots \mathrm{O}^{2-}$ & 985.87 & 0.3760 & 0.0 & 10 \\
& & & & 12 \\
\hline $\mathrm{O}^{2-} \ldots . \mathrm{O}^{2-}$ & 22764.00 & 0.1490 & 27.88 & 10 \\
\hline $\mathrm{Y}^{3+} \ldots \mathrm{O}^{2-}$ & 1345.10 & 0.3491 & 0.0 & \\
\hline
\end{tabular}

Table 2: Polarisable shell model parameters.

\section{Shell Model}




\begin{tabular}{|c|c|c|}
\hline Species & $\gamma / \mathrm{e}$ & $\mathrm{k} / \mathrm{eVA}^{-2}$ \\
\hline $\mathrm{O}^{2-}$ & -2.077 & 27.290 \\
\hline $\mathrm{Zr}^{4+}$ & 1.35 & 169.617 \\
\hline
\end{tabular}

In what follows we distinguish between "unrelaxed" structures, where all ions and vacancies reside on the ideal fluorite sites of $\mathrm{c}-\mathrm{ZrO}_{2}$, and "relaxed" structures in which the internal coordinates of the cell have been optimised. The structures are further split into two categories:

- Short-range, where the $\mathrm{O}_{\text {vac }}$ resides in the first O-coordination sphere of $\mathrm{Y}^{3+}$ (The so called, NN structures).

- Long-range, where the $\mathrm{O}_{\text {vac }}$ resides in the first O-coordination sphere of $\mathrm{Zr}^{4+}$ and second or greater O-coordination sphere of $\mathrm{Y}^{3+}$ (The so called NNN, NNNN or greater structures)

The defect formation energy $\left(\Delta \mathrm{E}_{\mathrm{f}}\right)$ is calculated relative to the formation energy per formula unit of c- $\mathrm{ZrO}_{2}\left(E_{\mathrm{ZrO}_{2}}\right)$ and that of the 40 atom cell of bixbyite $\mathrm{Y}_{2} \mathrm{O}_{3}\left(E_{\mathrm{Y}_{2} \mathrm{O}_{3}}\right)$ as:

$$
\Delta E_{f}=\frac{1}{n_{1}}\left(E_{\text {defect }}-n_{O} E_{Z r O_{2}}-n_{1} E_{Y_{2} O_{3}}\right)
$$

-equation 2

Where $\mathrm{E}_{\text {defect }}$ is the total energy of the supercell, $n_{0}$ the number of $\mathrm{ZrO}_{2}$ formula units, and $n_{1}$ is the number of $\mathrm{Y}_{2} \mathrm{O}_{3}$ substitutions in the supercell.

The DFT formation energy of an unrelaxed $\mathrm{Y}_{2} \mathrm{O}_{3}$ defect cluster was converged with respect to dopant concentration. YSZ was modelled at $14.3,6.7,3.2$, and $1.6 \mathrm{~mol} \%$ by introducing a symmetry equivalent $\mathrm{Y}_{2} \mathrm{O}_{3}$ defect cluster into 16, 32, 64 and 128 atom supercells of the conventional $\mathrm{c}-\mathrm{ZrO}_{2}$ cell respectively. Table 3 shows the formation energy of the defect cluster at different dopant concentrations. At $3.2 \mathrm{~mol} \%$ the formation energy converges to $10 \mathrm{meV}$, 
showing that this is the dilute limit and the point at which defect-defect interactions become negligible.

Table 3: Convergence of defect formation energies with respect to dopant concentration.

\begin{tabular}{|l|c|c|c|c|}
\hline Dopant concentration of $\mathrm{Y}_{2} \mathrm{O}_{3} / \mathrm{mol} \%$ & 14.3 & 6.7 & 3.2 & 1.6 \\
\hline DFT formation energy of unrelaxed $\mathrm{Y}_{2} \mathrm{O}_{3}$ defect / eV & 4.77 & 5.58 & 5.42 & 5.43 \\
\hline
\end{tabular}

A model of $3.2 \mathrm{~mol} \% \mathrm{YSZ}$ is created by introducing one $\mathrm{Y}_{2} \mathrm{O}_{3}$ unit into a 96 atom $2 \times 2 \times 2$ supercell of the conventional $\mathrm{c}-\mathrm{ZrO}_{2}$ cell. In this supercell, the high symmetry points of the first Brillouin zone of $\mathrm{c}-\mathrm{ZrO}_{2} \mathrm{X}, \mathrm{W}$ and $\mathrm{L}$ fold back onto $\Gamma$. This facilitates analysis of the imaginary phonon modes at the $\mathrm{X}$-point of $\mathrm{c}-\mathrm{ZrO}_{2}$ in terms of intra-cell atomic displacements. There are 63488 possible defect structures when introducing two substitutional $\mathrm{Y}^{3+}$ ions and one $\mathrm{O}_{\text {vac }}$ onto the ideal fluorite sites of the $2 \times 2 \times 2 \mathrm{c}-\mathrm{ZrO}_{2}$ supercell. Taking into account all translational and space-group symmetries this can be reduced to 28 symmetrically inequivalent structures ${ }^{61}$.

Correlations between data sets were analysed using regression analysis. Correlations are described by the coefficient of determination $\left(\mathrm{R}^{2}\right)$, which is the square of the Pearson correlation coefficient. The coefficient of determination has a value between 0 and 1 where 0 is no correlation and 1 is a perfect correlation.

\section{Born-Mayer-Huggins and Point Charge Models as Predictors of DFT Relaxation Energies}

The internal coordinates of the 28 symmetry inequivalent defect structures were fully relaxed using both the Born-Mayer-Huggins polarizable shell model, and DFT energy expressions. In Figure 1 the resulting relative defect formation energies are compared by plotting against a structure number that increases with increasing DFT defect formation energy. The model energies correlate poorly with the DFT energies, yielding an $\mathrm{R}^{2}$ of 0.35 . 


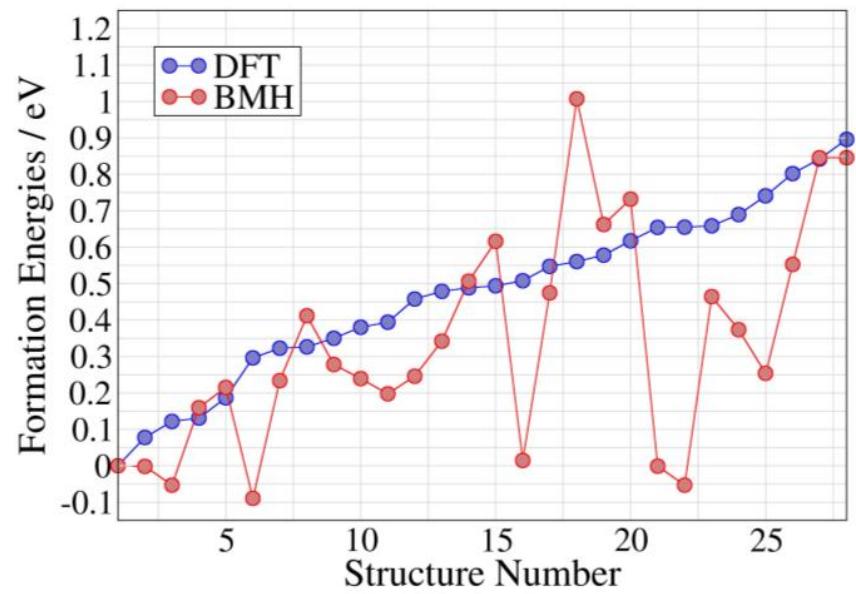

Figure 1: Defect formation energy relative to that of the lowest energy DFT structure for the 28 symmetry inequivalent structures calculated using the empirical BMH potential (red) and DFT (blue). The correlation between the two sets is low $\left(\mathrm{R}^{2}=0.35\right)$, indicating that the empirical potential reproduces DFT energy differences poorly.

The potential is unreliable in two respects. It fails to predict the same minimum energy defect structure as predicted by DFT, and predicts many structures to be significantly higher or lower in energy than their DFT equivalents. It also predicts many meta-stable structures to be unstable with respect to local reconstructions. These differences will be analysed in terms of the local geometries and the barriers to $\mathrm{O}_{\text {vac }}$ migration in the section titled: Analysis of Born-MayerHuggins Potential Model below.

The potential and DFT calculations do agree in predicting long-range NNN structures to be the lowest energy structures. The defect formation energy of the lowest energy structure computed by DFT (Figure 4) is $-1.55 \mathrm{eV}$. In this structure the $\mathrm{Y}^{3+}-\mathrm{O}_{\text {vac }}-\mathrm{Y}^{3+}$ defect cluster has an equilateral triangle topology, which is a common feature of all low energy structures.

Although much simpler than the Born-Mayer-Huggins potential, we also investigated an ionic model based on formal point charges with unrelaxed structures. Figure 2 shows the comparison 
between it and the DFT formation energies of the equivalent relaxed structures. There are two disjoint clusters of points within the scatter plot, which correspond exactly to the subsets of short-range and long-range defect geometries. All short range structures have rather high DFT formation energies and there is essentially no correlation between the point charge model and DFT. For these, the point charge model gives unphysically low formation energies. For long range structures, however, the point charge model correlates strongly to the DFT formation energies with an $\mathrm{R}^{2}$ value of 0.75 . We conclude that while the simple point charge model of unrelaxed geometries does not provide reliable total or relative energies for the defects it does provide a reliable predictor of the low energy defect arrangements.

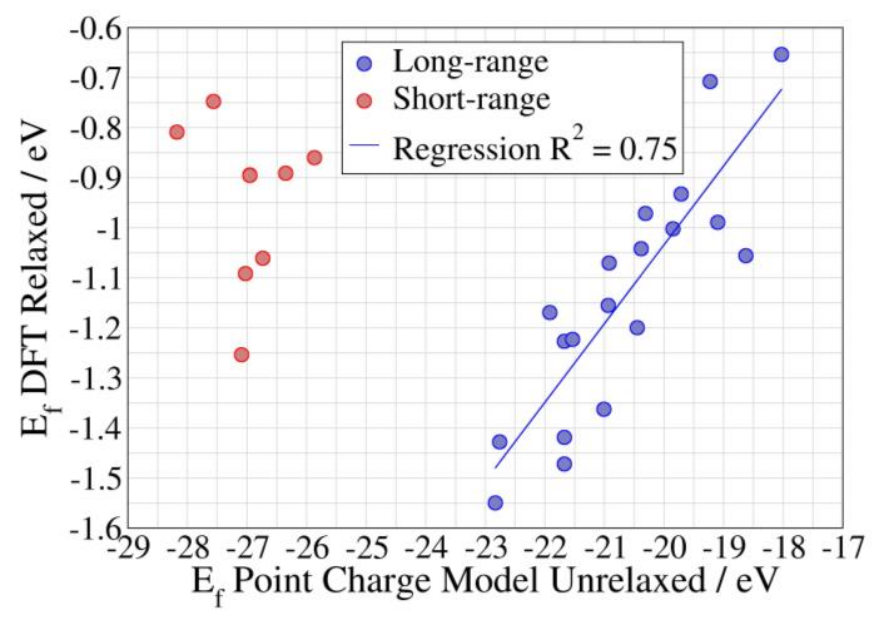

Figure 2: Electrostatic formation energies of the unrelaxed structures plotted against DFT formation energies of the relaxed structures. Blue points show the energetics of long-range structures, red points show the energetics of short-range structures.

\section{Distortion along Imaginary Phonon Modes as a Predictor of DFT Relaxation Energetics}

It is plausible that variation in structural relaxation around defects of different geometries is partly responsible for the variation in ordering energies. We investigate the relaxation energetics of our 28 DFT relaxed structures using a quantitative model of $a b$ initio force constants based on 
the unstable phonon mode of perfect, stoichiometric c- $\mathrm{ZrO}_{2}$. As can be seen in Figure 3, c- $\mathrm{ZrO}_{2}$ possesses two imaginary phonon modes at the $\mathrm{X}$ and $\mathrm{W}$-point of the first Brillouin zone. The phonon frequencies at the $\mathrm{X}$ and $\mathrm{W}$-points are calculated to be $i 217 \mathrm{~cm}^{-1}$ and $i 65 \mathrm{~cm}^{-1}$ respectively. The computed imaginary frequency at the X-point is similar to that reported in previous calculations using LDA and PBE functionals with projector augmented wave (PAW) and NCP pseudopotentials ${ }^{18-20,23}$. The frequency of the phonon at the W-point is more sensitive to numerical approximations, and for instance, varies from $i 18 \mathrm{~cm}^{-1}$ to $i 65 \mathrm{~cm}^{-1}$ as the FFT grid scale is increased from $\mathrm{x} 1.5$ to $\mathrm{x} 2.5$. This frequency was reported as approximately $40 \mathrm{~cm}^{-1}$ in previous PAW-PBE calculations using the Quantum ESPRESSO code ${ }^{20}$.

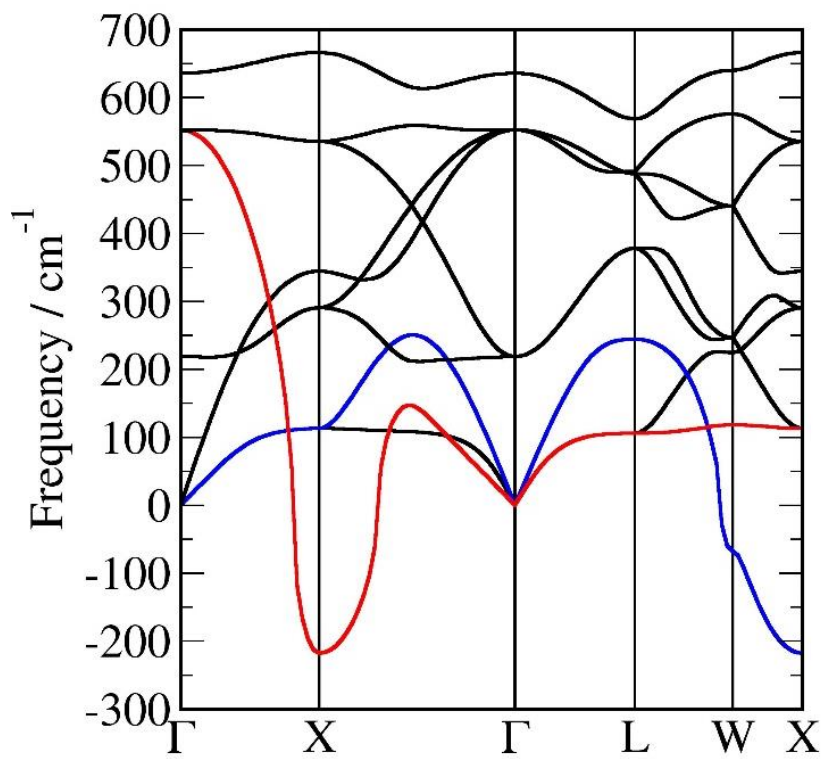

Figure 3: The computed phonon dispersion curves of $\mathrm{c}-\mathrm{ZrO}_{2}$ showing the imaginary frequency modes in the region of the $\mathrm{X}$ and W-points highlighted in red and blue.

The eigenvector of the X-point phonon corresponds to oxygen anions moving in the $\left\langle\begin{array}{lll}1 & 0 & 0\end{array}\right)$ direction with alternate ions along the $\left\langle\begin{array}{llll}0 & 1 & 0\end{array}\right\rangle$ direction moving in antiphase (Figure 4). The minimum energy configuration along this mode is $2 \mathrm{eV} /$ supercell lower in energy than c- $\mathrm{ZrO}_{2}$. In Figure 4 this displacement is compared with the relaxation observed in the lowest energy 
defect structure. The visible similarity strongly suggests that atomic displacement along this phonon eigenvector is responsible for relaxing the local strain induced by the dopant and vacancy.
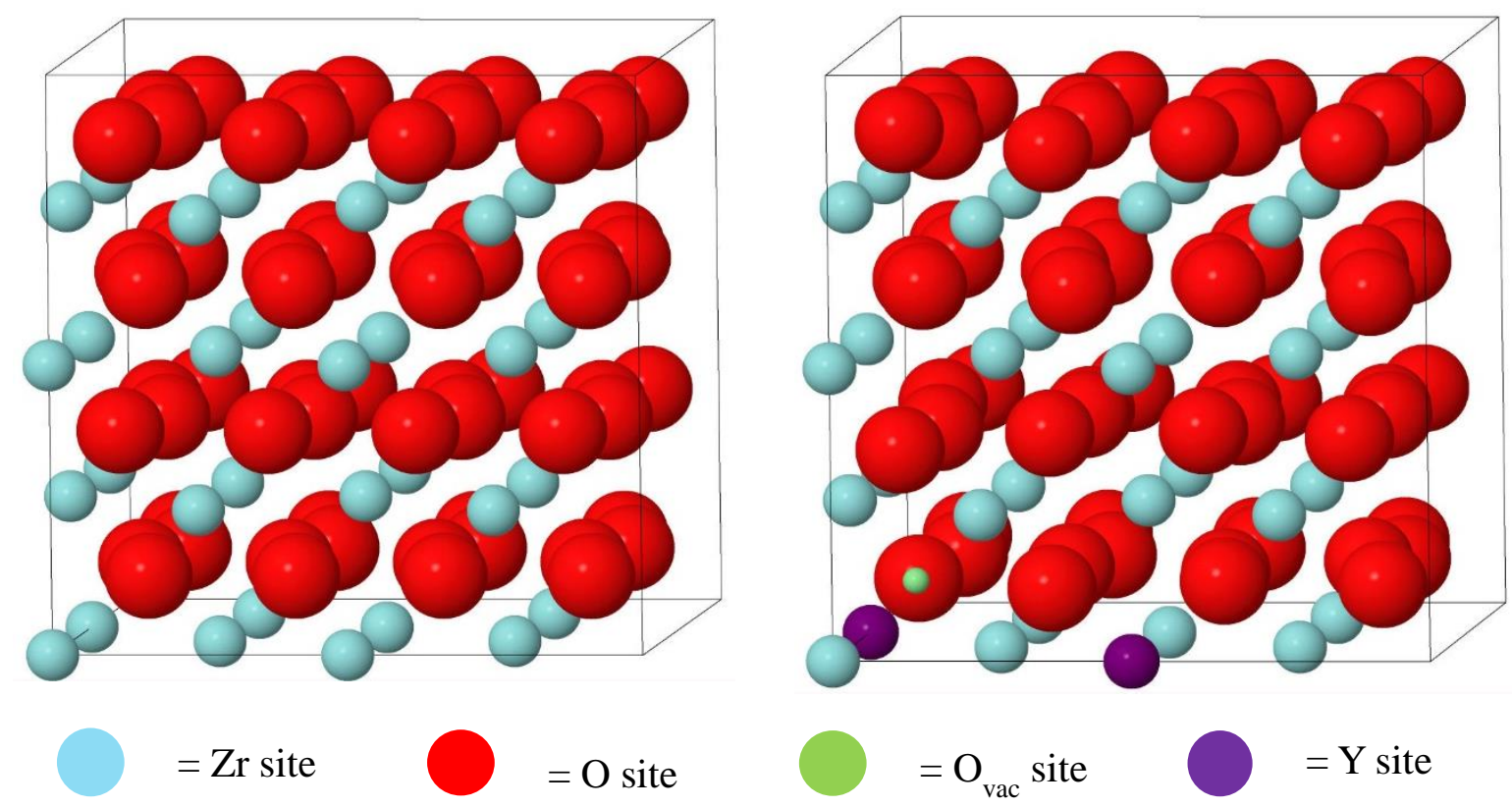

Figure 4: Left: relaxation of the oxygen anions pure along the imaginary X-point phonon mode in the $2 \times 2 \times 2$ supercell. Right: The relaxation pattern of the oxygen anions observed in the lowest energy defect structure. Note: the similarity of the relaxation patterns.

Relaxing the ideal bulk crystal along the imaginary W-point phonon eigenvector leads to displacement of the oxygen anions along a $\langle 210\rangle$ direction. The relaxation energy of the anions along the $\mathrm{W}$-point phonon is negligible compared to relaxation along the $\mathrm{X}$-point phonon, typically $10 \mathrm{meV} /$ supercell.

In Figure 5 the harmonic relaxation energy of the bulk X-point phonon is plotted against the formation energies of the relaxed structures computed using DFT. For each defect structure, $i$, the atomic displacements of relaxation are projected onto the bulk X-point phonon eigenvector to obtain an amplitude $\alpha_{i}$. The harmonic relaxation energy, in eV/cell, is then defined as: 


$$
\Delta E_{x}=\frac{1}{2} a^{2} \alpha_{i}^{2} \lambda_{x}
$$

where $a$ is the lattice constant of the unit cell, and $\lambda_{x}$ is the eigenvalue of the phonon mode.

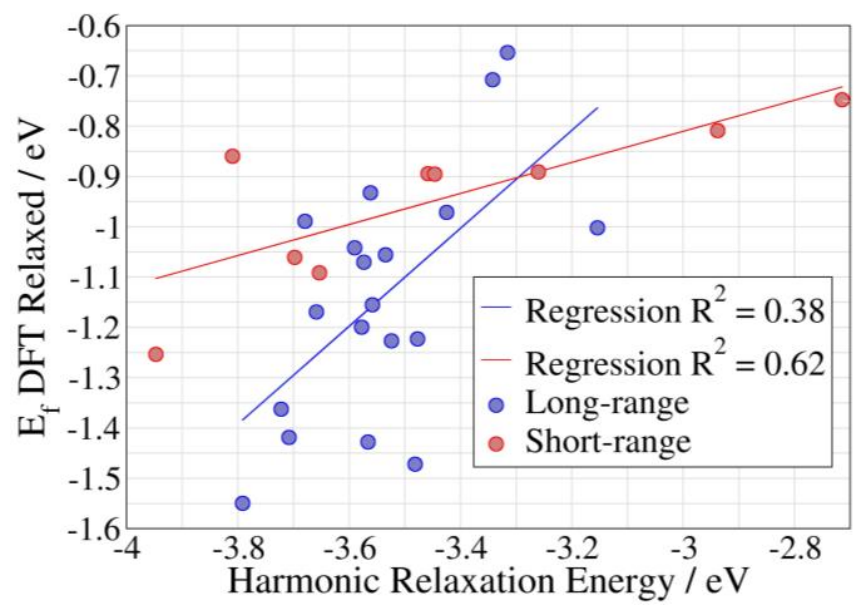

Figure 5: Relationship between the DFT formation energies of the relaxed structures and harmonic relaxation along imaginary X-point phonon modes. Blue points show the energetics of long-range structures, red points show the energetics of short-range structures.

For low energy structures and all long-range structures, the elastic relaxation energy along imaginary phonon modes is weakly correlated to the DFT energy of formation. Although the relaxation energy along the imaginary phonon modes is significant, it does not discriminate effectively between different structures. The correlation between the final relaxed DFT energies and the harmonic relaxation energy is poor with an $\mathrm{R}^{2}$ value of 0.38 . However the relative stability of high energy, short-range structures is more strongly correlated to the harmonic relaxation energy, with an $\mathrm{R}^{2}$ of 0.62 .

\section{Relative Importance of Electrostatic and Relaxation Energies}

The formation of $3.2 \mathrm{~mol} \% \mathrm{YSZ}$ isolated defect structures can be usefully viewed as a two-step process: firstly, the isolated $\mathrm{Y}_{2} \mathrm{O}_{3}$ unit is placed within the unrelaxed c- $\mathrm{ZrO}_{2}$ structure and 
secondly, the structure is allowed to relax. The relaxation is well described as a motion along the unstable X-point phonon mode with the unstable W-point mode making a negligible contribution. This suggests that relative defect formation energies can be predicted using an artificial synthesis model consisting of the unrelaxed electrostatic formation energies of the point charge model, and the harmonic relaxation along imaginary phonons, that is; ${ }^{62}$

$$
\Delta E_{f Y S Z}(i, R) \approx A \Delta E_{\text {elec }}(i, R)+B \Delta E_{\text {strain }}(\alpha, R)+C \quad \text {-equation } 4
$$

A, B and C are fitted constants and given below in Table 4, $\mathrm{E}_{\text {elec }}$ is the electrostatic formation energy of an unrelaxed defect structure in configuration $i$, and $\mathrm{E}_{\text {strain }}$ is the energy change from atomic relaxation along X-point imaginary phonons.

Through the synthesis model, the relative importance of electrostatics and imaginary phonons in determining the final energetic ordering of the relaxed DFT defect structures can be assessed. Figures 6 and 7 show the synthesis model energy plotted against the DFT formation energies of the relaxed long-range and short-range structures respectively. For long-range structures, almost all of the variation is accounted for by the electrostatic interaction of the defects. The coefficient of determination does not increase significantly when accounting for the X-phonon strain energy on top of the ionic energy (from 0.75 to 0.79 ). In contrast, the variation in short-range structures is described well by a combination of point charge electrostatics and relaxation along imaginary phonon modes. The coefficient of determination increases from 0.62 , with pure relaxation along imaginary X-point phonon modes, to 0.79 when taking into account electrostatics. To incorporate the two subcategories of structures into a single model, an understanding of a further 
destabilising force for short-range structures needs to be established. We do not currently have such an understanding.

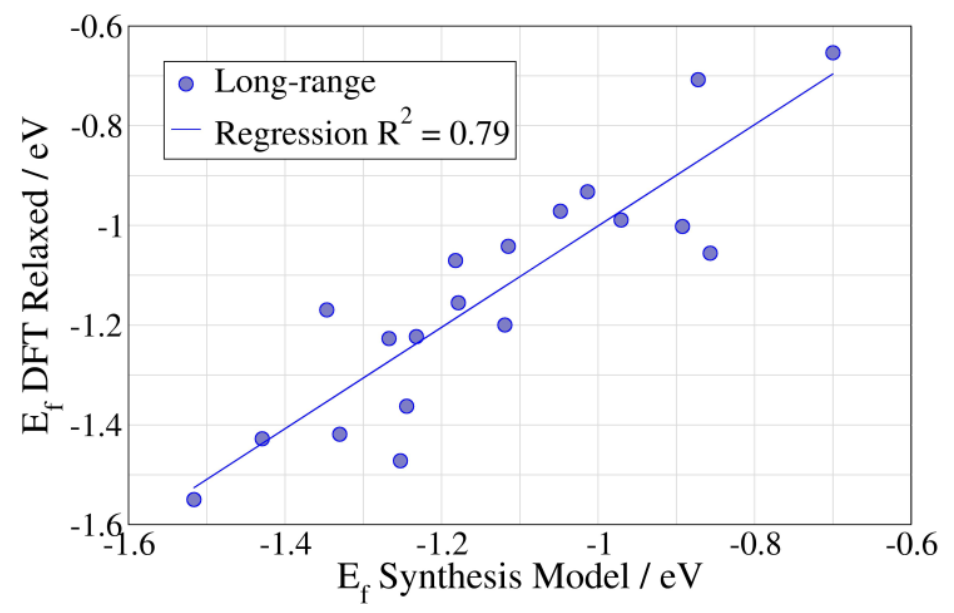

Figure 6: Long-range defect formation energies predicted by the synthesis model plotted against DFT formation energies of the relaxed long-range structures. The majority of the variation in the DFT energies is accounted for by electrostatic interactions.

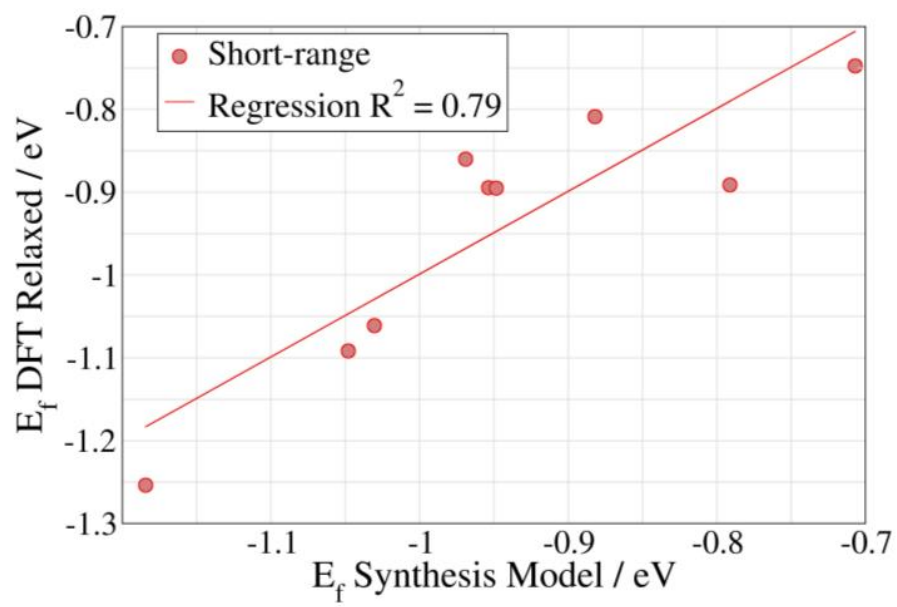

Figure 7: Short-range defect formation energies predicted by the synthesis model plotted against DFT formation energies of the relaxed short-range structures. The majority of the variation in the DFT energies is accounted for by relaxation along X-point imaginary phonon modes.

Table 4: Fitted constants for the synthesis models presented in Figures 6 and 7. 


\begin{tabular}{|l|c|c|c|}
\hline Fitted Constant & A (dimensionless) & B (dimensionless) & $\mathrm{C} / \mathrm{eV}$ \\
\hline Long-range & 0.14 & 0.34 & 2.89 \\
\hline Short-range & 0.13 & 0.44 & 3.97 \\
\hline
\end{tabular}

\section{Analysis of Born-Mayer-Huggins Potential Model}

As discussed in the introduction, the empirical potential model and DFT have been shown to predict the instability of many defect structures at 6.7 and $10.4 \mathrm{~mol} \%$. Unstable structures undergo barrier-less reconstructions of the $\mathrm{O}_{\text {vacs, }}$ where reconstructions predicted by DFT are often not predicted by the potential and vice versa ${ }^{44}$.

The final atomic positions of the relaxed structures are mapped back onto their unrelaxed geometries by identifying the ideal fluorite $\mathrm{O}$ and $\mathrm{Zr}$ sites closest to the location of the relaxed $\mathrm{Zr}, \mathrm{O}, \mathrm{Y}$, and $\mathrm{O}_{\mathrm{vac}}$ sites. It was found that for the potential model, seven structures were unstable and underwent barrier-less reconstructions of the $\mathrm{O}_{\mathrm{vac}}$ away from its initial position on the ideal crystal lattice. In contrast, DFT predicted one unstable structure with a flat energy surface with respect to $\mathrm{O}_{\text {vac }}$ reconstruction (pictured in Figure 8). Using DFT and a linear synchronous transit (LST) and quadratic synchronous transit (QST) maximisation transition state (TS) search, the energy barrier to reconstruction for the structure in Figure 8 was computed as $10 \mathrm{meV}$. This is small enough that thermally-activated reconstruction would be nearly instantaneous even at room temperature. 

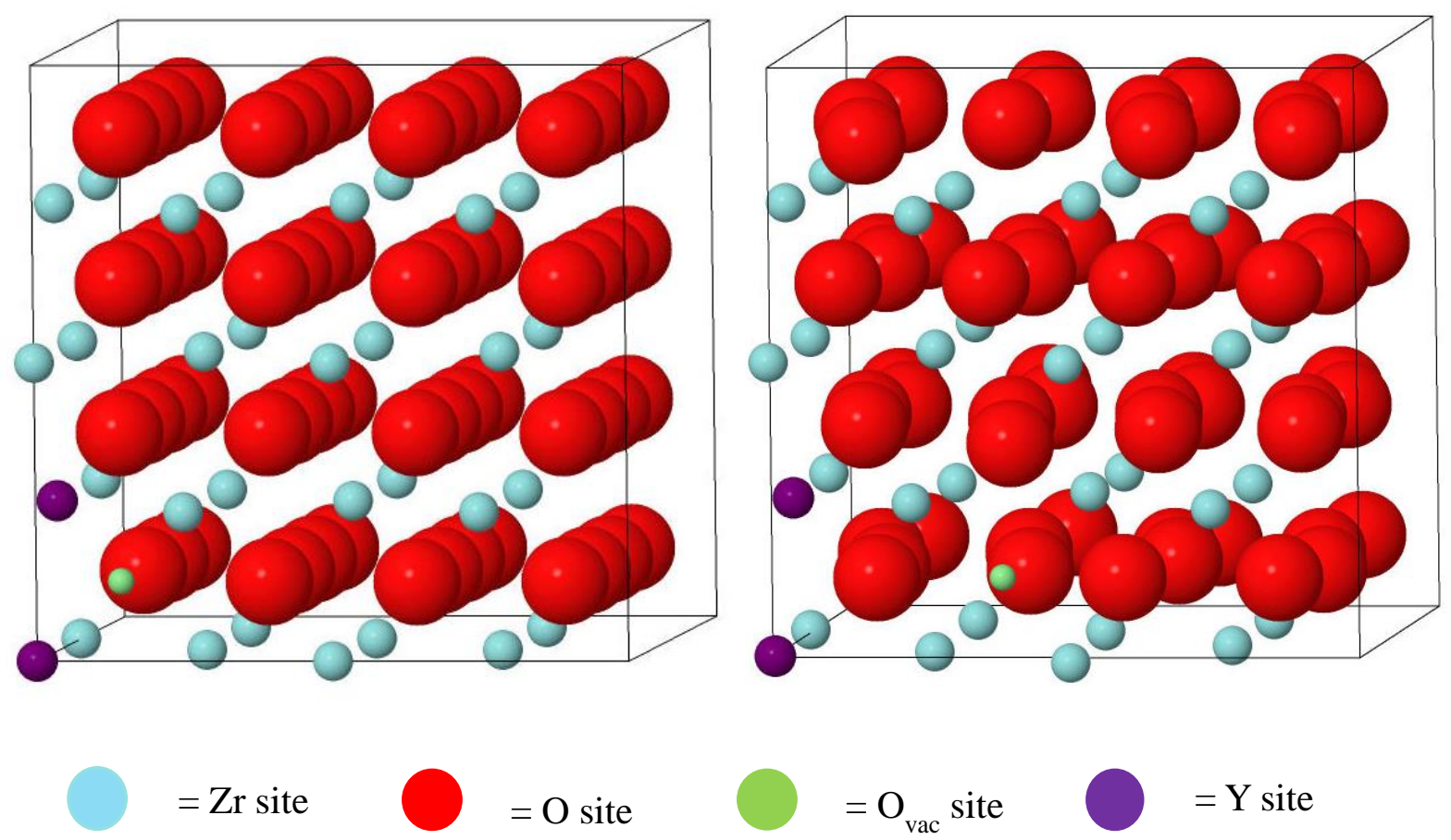

Figure 8: $\mathrm{O}_{\text {vac }}$ reconstruction observed upon strain relaxation in DFT Left: unrelaxed NN defect structure with two 7-fold O-coordinated $\mathrm{Y}^{3+}$ ions. Right: final strain relaxed NNN structure. The $\mathrm{O}_{\text {vac }}$ can be seem migrating between two metal tetrahedral sites. The energy barrier to reconstruction is calculated as $10 \mathrm{meV}$ using a DFT LST/QST TS search.

Through reconstructions, the majority of unstable structures predicted by the empirical potential relax under DFT to a geometry that can easily be mapped back to one of the 28 unrelaxed symmetry inequivalent structures. An example, with reference to Figure 1, is structure 16, which reconstructs to become similar to structure 1 . These structures have different unrelaxed geometries, and are meta-stable upon relaxation with DFT. The exception to this is structure 6. When relaxed, structure 6 takes on an intermediate geometry that cannot easily be mapped back onto one of the unrelaxed structures, with the $\mathrm{O}_{\text {vac }}$ residing between two ideal $\mathrm{O}^{2-}$ sublattice sites. Structure 6 is the lowest energy structure predicted by the potential model. When the 
intermediate geometry is relaxed in DFT, the $\mathrm{O}_{\text {vac }}$ spontaneously relaxes back to its original starting position on the $\mathrm{O}^{2-}$ sublattice.

The instability of structures within the potential model can be understood by considering anion migration pathways. Five of the unstable structures have an $\mathrm{O}_{\text {vac }}$ location such that a single $\mathrm{O}_{\text {vac }}$ migration leads to the $\mathrm{Y}^{3+}-\mathrm{O}_{\mathrm{vac}}-\mathrm{Y}^{3+}$ cluster taking on an $\mathrm{NNN}$ defect structure in equilateral triangle geometry. It must be the case that DFT predicts a barrier to $\mathrm{O}_{\text {vac }}$ migration while the potential does not. To estimate the DFT energy barriers to $\mathrm{O}_{\mathrm{vac}}$ migration, LST/QST - TS searches were performed on two structures that the potential predicts as unstable. Both structures are meta-stable in DFT, but can reconstruct to a more stable NNN structure through the single migration of an $\mathrm{O}_{\text {vac }}$. One structure starts as a short-range $\mathrm{NN}$ structure (structure 6), while the second starts as a long-range NNNN structure (structure 16). The energies of the transition states between structures 6 and 4, and structures 16 and 1 are calculated and presented in Table 5 .

Table 5: DFT calculated energy barriers to $\mathrm{O}_{\mathrm{vac}}$ migration.

\begin{tabular}{|l|l|l|l|}
\hline $\begin{array}{l}\text { Reconstruction } \\
\text { path }\end{array}$ & $\begin{array}{l}\text { Forward energy } \\
\text { barrier to TS / eV }\end{array}$ & $\begin{array}{l}\text { Reverse energy } \\
\text { barrier to TS / eV }\end{array}$ & $\begin{array}{l}\text { Total energy of } \\
\text { reconstruction / eV }\end{array}$ \\
\hline Structure $6 \rightarrow 4$ & 0.28 & 0.45 & -0.17 \\
\hline Structure $16 \rightarrow 1$ & 0.41 & 0.92 & -0.51 \\
\hline
\end{tabular}

The defect structure starting with NN geometry has a lower energy barrier to $\mathrm{O}_{\text {vac }}$ migration than the structure in NNNN geometry. The NNNN structure reconstructs to a significantly more stable energy minimum. The existence of energy barriers within DFT but not the potential can be understood by considering the local strain between $\mathrm{O}^{2-}$ ions and the metal sublattice. When an $\mathrm{O}_{\text {vac }}$ migrates, an $\mathrm{O}^{2-}$ ion must pass between two $\mathrm{Zr}^{4+}$ ions. The migration causes a distortion to 
the metal sublattice as the interatomic distance between the two $\mathrm{Zr}^{4+}$ ions increases to accommodate the movement. The size of the energy barrier appears to be linked to the distance the $\mathrm{Zr}^{4+}$ ions have to move from their ideal atomic sites. In the case of the NNNN to NNN migration, the interatomic distance of the $\mathrm{Zr}^{4+}$ ions increases from $3.98 \AA$ to $4.02 \AA$ in the TS state, before contracting $3.98 \AA$ in the final NNN structure. In the case of the NN to NNN migration, the interatomic distance increases from $3.97 \AA$ to $3.98 \AA$ in the TS state, and increases again to $4.0 \AA$ in the final NNN structure.

We conclude that the connectivity between local minima on the potential energy surface is poorly reproduced by the potential, suggesting that finite temperature properties and ion dynamics in particular are likely to be poorly reproduced by the potential model.

\section{Conclusion}

We have established atomistic geometries for the low energy isolated defect structures in $3.2 \mathrm{~mol} \% \mathrm{YSZ}$, and compared the accuracy and reliability of empirical potentials, simple electrostatics, and strain relaxation effects for predicting the DFT defect formation energies. We have proposed relatively easily computed chemical descriptors that can identify likely low energy defect structures of YSZ. We find that the best available empirical potential poorly recreates the general trend of increasing DFT formation energies across a series of 28 symmetry inequivalent structures. It also fails to reliably predict energy barriers associated with $\mathrm{O}_{\text {vac }}$ reconstruction when compared with DFT. In addition, the connectivity between defect structures topologies on the potential energy surface is poorly described.

Low energy defect structures have $\mathrm{NNN}$ type geometries where the $\mathrm{O}_{\text {vac }}$ resides in the second coordination sphere of a $\mathrm{Y}^{3+}$ ion and the first coordination sphere of a $\mathrm{Zr}^{4+}$ ion. This topology is 
optimal when the $\mathrm{Y}^{3+}-\mathrm{O}_{\text {vac }}-\mathrm{Y}^{3+}$ defect cluster has equilateral triangle geometry. A very good predictor of the likely low energy NNN structures, and indeed of all long-range NNNN and NNNNN type structures is the total electrostatic energy of a simple point charge model calculated at the unrelaxed geometries of the structures. However, the total electrostatic potential calculated at the unrelaxed geometries is a poor descriptor of short-range NN structures, where the $\mathrm{O}_{\text {vac }}$ resides in the first coordination shell of a $\mathrm{Y}^{3+}$ ion.

Local strain relaxation effects make significant contributions to the formation energy and can be mapped in terms of relaxation along the imaginary $\mathrm{X}$-point phonons of $\mathrm{c}-\mathrm{ZrO}_{2}$. Local strain relaxation is only significant in determining the energetic ordering of high energy structures with short-range interactions. All long-range structures exploit relaxation along imaginary phonon modes to a similar extent. The results suggest that defects can be characterised by two regimes: long-range structures, whose relative energetics correlate to, or can be predicted by point charge electrostatics; and short-range structures, whose relative energetics correlate to, or can be predicted by a model containing point charge electrostatics plus a harmonic relaxation energy along the imaginary X-point phonon modes of $\mathrm{c}-\mathrm{ZrO}_{2}$. Establishing an analytical description of a destabilising force at short-range will allow both regimes to be incorporated into a single model.

\section{Associated Content}

Attached with the manuscript are the CASTEP cell files for; c- $\mathrm{ZrO}_{2}, \mathrm{Y}_{2} \mathrm{O}_{3}$, and the 28 symmetry inequivalent defect structures at their unrelaxed and DFT relaxed geometries. The files are titled:

- $\quad \mathrm{c}-\mathrm{ZrO}_{2}$.cell

- $\mathrm{Y}_{2} \mathrm{O}_{3}$.cell 
- YSZ_X_unrelaxed.cell

- YSZ_X_relaxed.cell

$\mathrm{X}$ denotes the number of the structure and is equivalent to the structure number given in Figure 1. For a detailed description of the cell file format, readers are directed to the CASTEP website ${ }^{63}$. Documents containing the energetics of the parent materials and relaxed DFT defect structures are included, as well as the dynamical matrix of $\mathrm{c}-\mathrm{ZrO}_{2}$. These documents serve as a database for which future models can be developed. This material is available free of charge via the internet at http://pubs.acs.org.

\section{Author Information:}

Corresponding author: Michael.Parkes07@imperial.ac.uk

\section{Acknowledgements}

The authors would like to acknowledge the EPSRC for funding of this work, through a career acceleration fellowship, award number EP/I00422X/1. Images of atomic structures were prepared using $\mathrm{Jmol}^{64}$.

\section{References}

(1) Fergus, J. W. Electrolytes for Solid Oxide Fuel Cells. J. Power Sources 2006, 162 (1), 3040.

(2) Fergus, J. W. Green Chemistry and Chemical Engineering Solid Oxide Fuel Cells Materials Properties and Performance; 2009.

(3) Shishkin, M.; Ziegler, T. The Oxidation of $\mathrm{H} 2$ and $\mathrm{CH} 4$ on an Oxygen-Enriched YttriaStabilized Zirconia Surface: A Theoretical Study Based on Density Functional Theory. $J$. Phys. Chem. C 2008, 112 (49), 19662-19669. 
(4) Shishkin, M.; Ziegler, T. Oxidation of H2, CH4, and CO Molecules at the Interface between Nickel and Yttria-Stabilized Zirconia: A Theoretical Study Based on DFT. $J$. Phys. Chem. C 2009, 113 (52), 21667-21678.

(5) Shishkin, M.; Ziegler, T. Hydrogen Oxidation at the Ni/Yttria-Stabilized Zirconia Interface: A Study Based on Density Functional Theory. J. Phys. Chem. C 2010, 114 (25), 11209-11214.

(6) Cucinotta, C.; Bernasconi, M.; Parrinello, M. Hydrogen Oxidation Reaction at the Ni/YSZ Anode of Solid Oxide Fuel Cells from First Principles. Phys. Rev. Lett. 2011, 107 (20), 206103.

(7) Ammal, S. C.; Heyden, A. Combined DFT and Microkinetic Modeling Study of Hydrogen Oxidation at the Ni/YSZ Anode of Solid Oxide Fuel Cells. J. Phys. Chem. Lett. 2012, 3 (19), 2767-2772.

(8) Zhang, Y.; Fu, Z.; Wang, M.; Yang, Z. Oxygen Vacancy Induced Carbon Deposition at the Triple Phase Boundary of the Nickel/yttrium-Stabilized Zirconia (YSZ) Interface. $J$. Power Sources 2014, 261, 136-140.

(9) Shishkin, M.; Ziegler, T. Direct Modeling of the Electrochemistry in the Three-Phase Boundary of Solid Oxide Fuel Cell Anodes by Density Functional Theory: A Critical Overview. Phys. Chem. Chem. Phys. 2014, 16 (5), 1798-1808.

(10) Xia, X.; Oldman, R.; Catlow, R. Computational Modeling Study of Bulk and Surface of Yttria-Stabilized Cubic Zirconia. Chem. Mater. 2009, 21 (15), 3576-3585.

(11) Badwal, S. Zirconia-Based Solid Electrolytes: Microstructure, Stability and Ionic Conductivity. Solid State Ionics 1992, 52 (1-3), 23-32.

(12) Yashima, M.; Morimoto, K.; Ishizawa, N.; Yoshimura, M. Zirconia-Ceria Solid Solution Synthesis and the Temperature-Time-Transformation Diagram for the 1:1 Composition. $J$. Am. Ceram. Soc. 1993, 76 (7), 1745-1750.

(13) Ioffe, A. I.; Rutman, D. S.; Karpachov, S. V. On the Nature of the Conductivity Maximum in Zirconia-Based Solid Electrolytes. Electrochim. Acta 1978, 23 (2), 141-142.

(14) Kharton, V. V.; Naumovich, E. N.; Vecher, A. A. Research on the Electrochemistry of Oxygen Ion Conductors in the Former Soviet Union. I. ZrO 2 -Based Ceramic Materials. J. Solid State Electrochem. 1999, 3 (2), 61-81.

(15) Goff, J.; Hayes, W.; Hull, S.; Hutchings, M.; Clausen, K. Defect Structure of YttriaStabilized Zirconia and Its Influence on the Ionic Conductivity at Elevated Temperatures. Phys. Rev. B 1999, 59 (22), 14202-14219. 
(16) Bogicevic, A.; Wolverton, C.; Crosbie, G.; Stechel, E. Defect Ordering in Aliovalently Doped Cubic Zirconia from First Principles. Phys. Rev. B 2001, 64 (1).

(17) Bogicevic, A.; Wolverton, C. Nature and Strength of Defect Interactions in Cubic Stabilized Zirconia. Phys. Rev. B 2003, 67 (2).

(18) Zhao, X.; Vanderbilt, D. First-Principles Study of Structural, Vibrational, and Lattice Dielectric Properties of Hafnium Oxide. Phys. Rev. B 2002, 65 (23), 233106.

(19) Parlinski, K.; Li, Z.; Kawazoe, Y. First-Principles Determination of the Soft Mode in Cubic ZrO2. Phys. Rev. Lett. 1997, 78 (21), 4063-4066.

(20) Fadda, G.; Zanzotto, G.; Colombo, L. First-Principles Study of the Effect of Pressure on the Five Zirconia Polymorphs. I. Structural, Vibrational, and Thermoelastic Properties. Phys. Rev. B 2010, 82 (6), 064105.

(21) Fadda, G.; Zanzotto, G.; Colombo, L. First-Principles Study of the Effect of Pressure on the Five Zirconia Polymorphs. II. Static Dielectric Properties and Raman Spectra. Phys. Rev. B 2010, 82 (6), 064106.

(22) Negita, K. Lattice Vibrations and Cubic to Tetragonal Phase Transition in ZrO2. Acta Metall. 1989, 37 (1), 313-317.

(23) Ostanin, S.; Craven, A.; McComb, D.; Vlachos, D.; Alavi, A.; Paxton, A.; Finnis, M. Electron Energy-Loss near-Edge Shape as a Probe to Investigate the Stabilization of Yttria-Stabilized Zirconia. Phys. Rev. B 2002, 65 (22), 224109.

(24) Norberg, S. T.; Hull, S.; Ahmed, I.; Eriksson, S. G.; Marrocchelli, D.; Madden, P. A.; Li, P.; Irvine, J. T. S. Structural Disorder in Doped Zirconias, Part I: The Zr 0.8 Sc 0.2- X Y X O $1.9(0.0 \leq \mathrm{X} \leq 0.2)$ System. Chem. Mater. 2011, 23, 1356-1364.

(25) Norberg, S. T.; Hull, S.; Ahmed, I.; Eriksson, S. G.; Marrocchelli, D.; Madden, P. A.; Li, P.; Irvine, J. T. S. Structural Disorder in Doped Zirconias, Part I: The Zr 0.8 Sc 0.2- X Y X O $1.9(0.0 \leq \mathrm{X} \leq 0.2)$ System. Chem. Mater. 2011, 23 (6), 110223092625053.

(26) Marrocchelli, D.; Madden, P. a.; Norberg, S. T.; Hull, S. Structural Disorder in Doped Zirconias, Part II: Vacancy Ordering Effects and the Conductivity Maximum. Chem. Mater. 2011, 110223092559005.

(27) Pietrucci, F.; Bernasconi, M.; Di Valentin, C.; Mauri, F.; Pickard, C. EPR G-Tensor of Paramagnetic Centers in Yttria-Stabilized Zirconia from First-Principles Calculations. Phys. Rev. B 2006, 73 (13), 134112.

(28) Orera, V.; Merino, R.; Chen, Y.; Cases, R.; Alonso, P. Intrinsic Electron and Hole Defects in Stabilized Zirconia Single Crystals. Phys. Rev. B 1990, 42 (16), 9782-9789. 
(29) Azzoni, C.; Paleari, A. Sevenfold- and Sixfold-Coordinated Zr3+ Ions in Cubic Stabilized Zirconia: Crystal-Field Approach. Phys. Rev. B 1991, 44 (13), 6858-6863.

(30) Azzoni, C.; Bolis, L.; Paleari, A.; Samoggia, G.; Scardina, F. Disorder-Induced Optical and Paramagnetic Properties in Zirconium Dioxide: Role of Low-Symmetry Crystal Fields. Phys. Rev. B 1995, 51 (22), 15942-15946.

(31) Azzoni, C.; Paleari, A. Photoactivation of Hole Centers in Cubic Stabilized Zirconia. Phys. Rev. B 1996, 53 (1), 5-8.

(32) Merino, R.; Orera, V.; Lomonova, E.; Batygov, S. Paramagnetic Electron Traps in Reduced Stabilized Zirconia. Phys. Rev. B 1995, 52 (9), 6150-6153.

(33) Ben-Michael, R.; Tannhauser, D.; Genossar, J. ESR Centers in Reduced Stabilized Zirconia. Phys. Rev. B 1991, 43 (10), 7395-7404.

(34) D Steele and B E F Fender. The Structure of Cubic ZrO 2 :YO 1.5 Solid Solutions by Neutron Scattering. J. Phys. C Solid State Phys. 1974, 7 (1), 1.

(35) Roth, W.; Wong, R.; Goldman, A.; Canova, E.; Kao, Y.; Dunn, B. Structure of Additives in B"-Alumina and Zirconia Superionic Conductors. Solid State Ionics 1986, 18-19, $1115-$ 1119.

(36) Morikawa, H.; Shimizugawa, Y.; Marumo, F.; Harasawa, T.; Ikawa, H.; Tohji, K.; Udagawa, Y. Local Structures Around Y Atoms in Y2O3-Stabilized Tetragonal ZrO2. J. Ceram. Soc. Japan 1988, 96 (1111), 253-258.

(37) Tuilier, M. H.; Dexpert-Ghys, J.; Dexpert, H.; Lagarde, P. X-Ray Absorption Study of the $\mathrm{ZrO} 2 \square$ Y2O3 System. J. Solid State Chem. 1987, 69 (1), 153-161.

(38) Li, P.; Chen, I.-W.; Penner-Hahn, J. X-Ray-Absorption Studies of Zirconia Polymorphs. II. Effect of Y2O3 Dopant on ZrO2 Structure. Phys. Rev. B 1993, 48 (14), 10074-10081.

(39) Catlow, C. R. A.; Chadwick, A. V.; Greaves, G. N.; Moroney, L. M. EXAFS Study of Yttria-Stabilized Zirconia. J. Am. Ceram. Soc. 1986, 69 (3), 272-277.

(40) Cole, M.; Catlow, C. R. A.; Dragun, J. P. EXAFS Studies of Doped-ZrO2 Systems. J. Phys. Chem. Solids 1990, 51 (6), 507-513.

(41) Kawata, K.; Maekawa, H.; Nemoto, T.; Yamamura, T. Local Structure Analysis of YSZ by Y-89 MAS-NMR. Solid State Ionics 2006, 177 (19-25), 1687-1690.

(42) Stapper, G.; Bernasconi, M.; Nicoloso, N.; Parrinello, M. Ab Initio Study of Structural and Electronic Properties of Yttria-Stabilized Cubic Zirconia. Phys. Rev. B 1999, 59 (2), 797-810. 
(43) Dalach, P.; Ellis, D. E.; van de Walle, A. First-Principles Thermodynamic Modeling of Atomic Ordering in Yttria-Stabilized Zirconia. Phys. Rev. B 2010, 82 (14), 144117.

(44) Pietrucci, F.; Bernasconi, M.; Laio, A.; Parrinello, M. Vacancy-Vacancy Interaction and Oxygen Diffusion in Stabilized Cubic ZrO2 from First Principles. Phys. Rev. B 2008, 78 (9), 094301.

(45) Brinkman, H. W.; Briels, W. J.; Verweij, H. Molecular Dynamics Simulations of YttriaStabilized Zirconia. Chem. Phys. Lett. 1995, 247 (4-6), 386-390.

(46) Dwivedi, A.; Cormack, A. N. A Computer Simulation Study of the Defect Structure of Calcia-Stabilized Zirconia. Philos. Mag. A 1990, 61 (1), 1-22.

(47) Lewis, G. V; Catlow, C. R. A. Potential Models for Ionic Oxides. J. Phys. C Solid State Phys. 1985, 18 (6), 1149-1161.

(48) Khan, M. S.; Islam, M. S.; Bates, D. R. Cation Doping and Oxygen Diffusion in Zirconia: A Combined Atomistic Simulation and Molecular Dynamics Study. J. Mater. Chem. 1998, 8 (10), 2299-2307.

(49) Kanzaki, H. Point Defects in Face-Centred Cubic lattice-I Distortion around Defects. $J$. Phys. Chem. Solids 1957, 2 (1), 24-36.

(50) Clark, S. J.; Segall, M. D.; Pickard, C. J.; Hasnip, P. J.; Probert, M. J.; Refson, K.; Payne, M. C. First Principles Methods Using CASTEP. Zeitschrift für Krist. 2005, 220 (5-6), $567-570$.

(51) Gale, J. D. GULP: A Computer Program for the Symmetry-Adapted Simulation of Solids. J. Chem. Soc. Faraday Trans. 1997, 93 (4), 629-637.

(52) Perdew, J. P.; Burke, K.; Ernzerhof, M. Generalized Gradient Approximation Made Simple. Phys. Rev. Lett. 1996, 77 (18), 3865-3868.

(53) Vanderbilt, D. Soft Self-Consistent Pseudopotentials in a Generalized Eigenvalue Formalism. Phys. Rev. B 1990, 41 (11), 7892-7895.

(54) Refson, K. Variational Density-Functional Perturbation Theory for Dielectrics and Lattice Dynamics. Phys. Rev. B 2006, 73 (15), 155114.

(55) Ramer, N.; Rappe, A. Designed Nonlocal Pseudopotentials for Enhanced Transferability. Phys. Rev. B 1999, 59 (19), 12471-12478.

(56) Rappe, A. Opium: Pseudopotential generation project, http://opium.sourceforge.net/index.html http://opium.sourceforge.net/index.html (accessed Sep 5, 2014). 
(57) Monkhorst, H. J.; Pack, J. D. Special Points for Brillouin-Zone Integrations. Phys. Rev. B 1976, 13 (12), 5188-5192.

(58) Terki, R.; Bertrand, G.; Aourag, H.; Coddet, C. Structural and Electronic Properties of Zirconia Phases: A FP-LAPW Investigations. Mater. Sci. Semicond. Process. 2006, 9 (6), 1006-1013.

(59) Badehian, H. A.; Salehi, H.; Ghoohestani, M. First-Principles Study of Elastic, Structural, Electronic, Thermodynamical, and Optical Properties of Yttria (Y 2 O 3 ) Ceramic in Cubic Phase. J. Am. Ceram. Soc. 2013, 96 (6), 1832-1840.

(60) Segall, M. D.; Lindan, P. J. D.; Probert, M. J.; Pickard, C. J.; Hasnip, P. J.; Clark, S. J.; Payne, M. C. First-Principles Simulation: Ideas, Illustrations and the CASTEP Code. $J$. Phys. Condens. Matter 2002, 14 (11), 2717-2744.

(61) Parkes, M.; Refson, K.; d'Avezac, M.; Offer, G.; Brandon, N. P.; Harrison, N. Determining Surface Chemistry and Vibrational Properties of SOFC Anode Materials Through Ab Initio Calculations. ECS Trans. 2013, 57 (1 ), 2419-2427.

(62) Shchyglo, O.; Díaz-Ortiz, A.; Udyansky, A.; Bugaev, V. N.; Reichert, H.; Dosch, H.; Drautz, R. Theory of Size Mismatched Alloy Systems: Many-Body Kanzaki Forces. $J$. Phys. Condens. Matter 2008, 20 (4), 045207.

(63) CASTEP cell keywords and data blocks http://www.tcm.phy.cam.ac.uk/castep/documentation/WebHelp/html/keywords/k_main_st ructure.htm (accessed Aug 20, 2014).

(64) Jmol: an open-source Java viewer for chemical structures in 3D. http://www.jmol.org/ (accessed Aug 19, 2014).

\section{Table of Contents Graphic:}

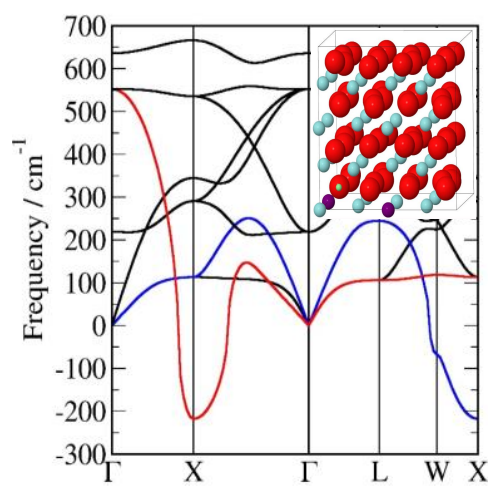

